\title{
Prevalence of human papilloma virus infections among women with systemic lupus erythematosus
}

\author{
Poopak Mohaghegh ${ }^{1}$, Bahareh Hamedi ${ }^{2}$, Saeedeh Shenavandeh ${ }^{3 *}$, Akbar Safaei ${ }^{4}$, Mohammadali Nazarinia ${ }^{2,5}$, \\ Elham Aflaki ${ }^{3}$, Zahra Habibagahi ${ }^{3}$, Behnaz Valibeigi ${ }^{4}$ \\ ${ }^{1}$ Department of Internal Medicine, Fasa University of Medical Sciences, Fasa, Iran; ${ }^{2}$ Department of Obstetrics and Gynecology, Shiraz \\ University of Medical Sciences, Shiraz, Iran; ${ }^{3}$ Department of Internal Medicine, Division of Rheumatology, Shiraz. University of \\ Medical Sciences, Shiraz, Iran; ${ }^{4}$ Department of Pathology, Shiraz University of Medical Sciences, Shiraz, Iran; ${ }^{5}$ Shiraz Geriatric \\ Research Center, Shiraz University of Medical Sciences, Shiraz, Iran
}

\begin{abstract}
Systemic lupus erythematosus (SLE) is considered one of the risk factors for cervical cytological abnormalities and high-risk HPV infection. However, there is a scarcity of data about the prevalence of high-risk HPV infection in patients with lupus. In order to define strategies for cancer prevention, we aimed to determine the prevalence of HPV infections in women with SLE compared to a control group. Fifty patients with SLE from the SLE clinic in Hafez Hospital, Shiraz University of Medical Sciences, were compared to 50 healthy married control women. In both groups, routine Pap smears were obtained, and the endocervical side of the spatula was sent for HPV DNA analysis. The case and control groups were compared for the presence and type of HPV infection as well as the relation between its presence with immunosuppressant use and disease activity. HPV infection was detected in 4\% (2.50) of lupus patients, but it was not seen in the control group. HPV typing identified types 16 and 18 in these two patients. No relationship between HPV infection and immunosuppressant use or activity of disease was seen in the current study. There was no statistically significant difference in HPV prevalence between patients and the control group $(P=0.495)$. Results of this study showed that SLE and the use of immunosuppressants in patients with lupus were not risks for high-risk HPV infection or cervical dysplasia in our area compared to the normal population, but further studies on more patients receiving immunosuppressants and biologics are recommended.
\end{abstract}

Keywords: human papillomavirus, Iran, prevalence, systemic lupus erythematosus.

\section{Introduction}

Systemic lupus erythematosus (SLE) is a disease caused by an abnormal autoimmune response, and anti-inflammatory and/or immunosuppressive drugs are needed for its treatment [1]. In SLE, it has been shown that factors like genetic and ethnic background, immunological abnormalities, extent of organ damage, and behavioral patterns can increase the susceptibility of patients to human papillomavirus (HPV) infection and cervical cytological abnormalities [2].

More than 80 types of HPV have been characterized. HPV DNA is present in $80 \%$ to $90 \%$ of squamous cell carcinomas and adenocarcinomas of the cervix worldwide; it is also found in cervical intraepithelial neoplasia (CIN). Genotype 16, commonly seen in squamous cell carcinoma, and genotype 18, seen more in adenocarcinoma, are classified as carcinogens [3].

Some studies have reported that the rate of cervical dysplasia as a result of the presence of high-risk HPV was increased in woman with lupus; also, the increased prevalence of HPV may be due to exposure to immunosuppressive drugs like cyclophosphamide [4]. It was not clear whether these associations were host-related, disease-related, or, alternatively, related to drug effects [5].

Different results were obtained by different studies for the prevalence of high-risk HPV infection and cervical cytological abnormalities in patients with lupus based on their ethnicity and genetics [2, 3, 6]. Studies on the prevalence of high-risk HPV infection using PCR and cervical cytological abnormalities in SLE patients of different ethnic backgrounds and regions are limited. Each country needs to know the local prevalence rate of HPV in

\footnotetext{
* Corresponding Author: Saeedeh Shenavandeh, E-mail: shenavande@ sums.ac.ir, Tel/Fax: +98 71 36474316, Po Box: 71345-1414 Received: 01 December 2017; Accepted: 12 February 2018
} 
order to define its strategies for cancer prevention and future vaccination. Concerns still exist about the risk of vaccination for HPV in patients with autoimmune diseases, although the benefits seem clear [7].

In this study, we aimed to determine the prevalence of HPV infections in southern Iranian women with SLE and its relationship with immunosuppressive therapies.

\section{Materials and Methods}

Fifty married SLE patients from the SLE clinic in Hafez Hospital who met the ACR criteria for SLE disease [8] were selected by convenience and sequential sampling methods, and an analytical cross-sectional study was done on them; 50 healthy control women from healthy married candidates in Shiraz city referring to the gynecology clinic were enrolled as the control group. Patients and the healthy control group were examined and sampled from September, 2011 until September, 2012. They were matched for age.

Patients were included in this study if they were sexually active. Patients who had never had intercourse and were younger than 20 years of age were excluded.

Demographic information (age, marital status, and tobacco use) and reproductive and sexual history (number of pregnancies, parity, and number of sexual partners) were recorded on a screening form after patients gave their consent. Disease duration, extent of organ involvement, and types of treatment were included as well.

The selected patients who fit the criteria and signed the consent form and the healthy control group were sent to the gynecology clinic of Hafez Hospital and were examined by a gynecologist. Routine Pap smears were obtained with a spatula, rolled onto a microscope slide and spray-fixed, and the endo-cervical side of the spatula was swirled in $1 \mathrm{ml}$ of sterile phosphate buffered saline and lysis buffer solution to release the remaining cells for analysis of HPV DNA. The samples were transported and stored at $-20^{\circ} \mathrm{C}$ prior to further analyses. A pathologist evaluated the cervical smears to detect any cytological abnormalities. The cervical smears were reported according to the Bethesda Classification System [6]. The frozen samples were processed for polymerase chain reaction to check for HPV.

\section{HPV detection}

Nucleic acids were extracted with the QIAamp DNA extraction kit (Qiagen, Hilden, Germany) according to the kit's instructions. Assays with PCR to detect 317-bp b-actin DNA were performed with forward primer 5'ATCATGTTTGAGACCTCCAA-3' and reverse primer 5'-CATCTCTTGCTCGAAGTCCA-3' for each processed specimen to assess DNA integrity. A GP5+/GP6+ primer set (Metabion International, Martinsried, Germany) was used to amplify a 140-bp fragment of the common region in all HPV with 5'-TTTGTTACTGTGGTAGATACT-3' as the forward primer and 5'GAAAAATAAACTGTAAATCATATT- $3^{\prime}$ as the reverse primer.

The 25- $\mu$ l mixture for the PCR reaction included approximately $100 \mu \mathrm{g}$ extracted DNA, $0.2 \mathrm{mM}$ dNTP mix (Fermentas, Litvani), 1 U Taq DNA polymerase (Fermentas), $2.5 \mu \mathrm{l} 10$ PCR buffer, and $20 \mu \mathrm{m}$ of each primer (TIB Molbiol, Berlin, Germany). Thermocycler conditions were initial denaturation at $94^{\circ} \mathrm{C}$ for $3 \mathrm{~min}$, followed by 45 cycles of denaturation at $94^{\circ} \mathrm{C}$ for $30 \mathrm{~s}$, annealing at $50^{\circ} \mathrm{C}$ for $60 \mathrm{~s}$, and extension at $72^{\circ} \mathrm{C}$ for $30 \mathrm{~s}$, with a final extension at $72^{\circ} \mathrm{C}$ for $5 \mathrm{~min}$. The PCR were analyzed by electrophoresis on $2.5 \%$ agarose gel. A 50-bp range molecular weight ladder and appropriate negative and positive controls were done at the same time.

\section{HPV genotyping}

The HPV-positive samples were then checked for HPV genotyping for genotypes 16 and 18. Forward primer 5'TCAAAAGCCACTGTGTCCTG-3' and reverse primer 5'-CGTGTTCTTGATGATCTGCA-3' were used to amplify HPV-16 DNA, and forward primer 5'GACACATTGGAAAAACTA AC- $3^{\prime}$ and reverse primer 5'-TAG TGC CCA GCT ATG TTG TG -3' were used for HPV 18. PCR reactions were carried out in a total volume of $25 \mu \mathrm{l}$ containing $2.5 \mu \mathrm{l} 10 \mathrm{PCR}$ buffer, $1.5 \mathrm{mM} \mathrm{MgCl}_{2}$, $0.2 \mathrm{mM}$ of each dNTP (Fermentas), $1 \mathrm{U}$ Taq DNA polymerase (Fermentas, Litvani), and $20 \mathrm{pmol}$ of each primer (a mixture of HPV-16 and HPV-18-specific primers) (TIB Molbiol). Thermocycler conditions were initial denaturation at $94^{\circ} \mathrm{C}$ for three minutes and then by 30 cycles of denaturation at $94^{\circ} \mathrm{C}$ for one minute, annealing at $58^{\circ} \mathrm{C}$ for one minute, and extension at $72^{\circ} \mathrm{C}$ for one minute, with final extension at $72^{\circ} \mathrm{C}$ for five minutes. The amplified fragments were visualized by electrophoresis on $2.5 \%$ agarose gels and stained with GelRed.

\section{Statistical analysis}

Clinical parameters are presented as mean \pm SD. The SLE patients and the controls were compared by $\chi^{2}$ test. Statistical analysis was done using SPSS 19.

\section{Results}

\section{Characteristics of patients and controls}

SLE patients and controls were all Iranian from both rural and urban areas. The mean age was $35.7 \pm 4.88$ years in lupus patients and $36.6 \pm 7.196$ years in the control group. Sixty-four percent of the SLE patients and $78 \%$ of the controls were under 39 years of age. No significant 
difference between these two groups was observed (Table $1)$.

Table 1. Age distribution of patients and controls

\begin{tabular}{lll}
\hline Age group (\%) & SLE $(\mathbf{n = 5 0 )}$ & Control $(\mathbf{n = 5 0 )}$ \\
\hline 20-29year & $8(16 \%)$ & $12(24 \%)$ \\
30-39year & $24(48 \%)$ & $27(54 \%)$ \\
40-49year & $17(34 \%)$ & $10(20 \%)$ \\
$\geq 50$ year & $1(2 \%)$ & $1(2 \%)$ \\
\hline Age (mean \pm SD) years & $35.7 \pm 4.88$ & $36.6 \pm 7.196$ \\
p-value: 0.465 & & \\
\hline
\end{tabular}

The mean disease duration of SLE patients was 7.52 years (range $=1-20$ years). Major organ involvement (central nervous system and kidney) was seen in $34 \%$ (17.50) of patients. The mean SLE disease activity index (SLEDAI) was $0.44 \pm 1.07$.

Drugs recently used by patients with lupus were prednisolone $<10 \mathrm{mg}$ in $45(90 \%)$ patients, prednisolone $>10 \mathrm{mg}$ in 2 (4\%), Hydroxychloroquine in $35(70 \%)$, Azathioprine in $5(10 \%)$, Cyclosporine in $6(12 \%)$, Mycophenolate mofetil in $5(10 \%)$, Cyclophosphamide in $2(4 \%)$, and methotrexate in $4(8 \%)$ of patients.

Two (4\%) of the patients were receiving CTX during the evaluation in this study, but $28 \%$ (14.50) of them had previously received CTX during the course of their disease that was not included in the current study.

There were no smokers among the controls or the patients. All patients and controls had one sexual partner. Mean SLE damage index at the time of gynecologic evaluation was $0.32 \pm 0.62$. Mean SLE activity index was $0.44 \pm 1.07$.

The pathologist did not report any dysplasia or neoplasia in the Pap smears of the patients or controls. Pap smears were evaluated for inflammatory or other pathologic changes in 50 SLE patients (Table 2). Comparisons between these two groups showed no statistically significant difference; therefore, no relationship between consumption of immunosuppressive medications and inflammatory or pathologic changes in Pap smears was observed.

Table 2. Percentage of pap smears with inflammatory changes (\% of total) in SLE patients related to immunosuppressive medication

\begin{tabular}{ccccc}
\hline & $\begin{array}{c}\text { SLE patients } \\
\text { who received } \\
\text { immunosuppre } \\
\text { ssive } \\
\text { medications } \\
\text { (N:22) }\end{array}$ & $\begin{array}{c}\text { SLE patients } \\
\text { who never } \\
\text { received } \\
\text { immunosuppress } \\
\text { ive medications } \\
\text { (N:28) }\end{array}$ & $\begin{array}{c}\text { Total } \\
\text { (N:50) }\end{array}$ & $\begin{array}{c}\text { P } \\
\text { value }\end{array}$ \\
\hline $\begin{array}{c}\text { Number } \\
\text { of pap } \\
\text { smears } \\
\text { with }\end{array}$ & & & & \\
$\begin{array}{c}\text { inflamm } \\
\text { atory } \\
\text { changes } \\
(\%)\end{array}$ & $8(16 \%)$ & $10(20 \%)$ & $18(36 \%)$ & 0.716 \\
\hline
\end{tabular}

HPV infection was detected in $2(4 \%)$ of the patients with SLE, but it was not seen in the control group. There was no statistically significant difference between the patients and the control group in HPV prevalence $(P=0.495)$. HPV typing assays showed types 16 and 18 in these two patients. One of them had received Cyclophosphamide in her disease course, but another was not on immunosuppressive drugs; therefore, there was no relationship between HPV infection and immunosuppressive medications in the current study. There was no evidence of inflammatory changes in the Pap smears of these two patients. One of these patients had lupus nephritis with SLAEDAI: 4 during the study, and one of them had silent disease with no activity and SLEDAI:0 and was only receiving hydroxychloroquine and low-dose prednisolone therapy. In both patients, the damage index was 0 , so there was no correlation between presence of HPV infection and disease activity. It is noteworthy that this study had a low prevalence of positivity for HPV infection; a higher number of patients should be evaluated in future studies to investigate the relationship between this infection and disease activity.

\section{Discussion}

In this study, HPV infection and Pap smear changes in patients with SLE and a normal population were compared and searched for increasing risk in patients receiving immunosuppressive drugs. Moreover, because some genotypes (like genotypes 16 and 18) are classified as carcinogens, HPV typing was done to find the most common types of HPV in the patients of this study. In some studies, SLE was considered a risk factor for cervical malignancy and high-risk HPV infection [4]. The results of the current study showed that the presence of SLE and receiving immunosuppressants were not risks for high risk HPV infection or abnormal Pap smears. The prevalence of HPV in Shiraz was reported to be $5.5 \%$ in 2008; this figure is similar to the current findings in patients with SLE in 2011-2012. Although the control group in this study had no HPV infections, the number of participants in the control group was low [3].

The prevalence of HPV infection seems to be different according to worldwide epidemiological studies. It has been reported that HPV prevalence in women with normal cervical cytology is about $1.4-25.6 \%[9,10]$.

An IARC survey in Asia detected 9.6\% HPV positive patients, of which $5.4 \%$ had high-risk types. HPV-16 was two times more than other high-risk types in all regions (Iran was not included in their study) [3].

In Iran, the prevalence of HPV was different in multiple areas studied. In Zabol, Iran, it was considerably high at 
$29 \%$ and $37.9 \%$ in monogamous and polygamous women, respectively. The majority of HPV infections were reported in the age group of 15-25 years. HPV-18 was observed more than other types in the monogamous group [11]. In another study conducted in the University Center, Shiraz, Fars, Iran, on 402 healthy women with normal Pap smears from July to December, 2008, the prevalence of positive HPV was 5.5\%. High-risk HPV type 16 was detected in $2 \%$ of women, and no patient had HPV-18 [3].

In a study by Tam et al., among 85 SLE patients, HPV was reported in $4.7 \%$, but this rate was $1.1 \%$ in 2080 healthy subjects. In their study, patients with SLE had a greater number of reports of abnormal Pap smears than the controls, even with adjustments for HPV status. Immunosuppressant usage increased the susceptibility to high-risk HPV infection, but it did it not correlate with abnormal Pap smears [12].

In 2007 in the United Kingdom, Rahul Nath et al. investigated patients with recent diagnoses of lupus compared to the normal population and showed a higher rate of HPV infections (especially the European HPV-16 variants), abnormal cervical cytology, and squamous intraepithelial lesions in their lupus patients [5]. In another review study in 2011, lupus was not found to be a risk for cervical cancer; it was, however, recommended that lupus patients be considered high risk for HPV infection and cervical dysplasia compared to control groups [6].

In Korea, $24.6 \%$ of 134 patients with lupus had highrisk HPV infections compared with $7.9 \%$ in 4595 healthy subjects $(P<0.001)$. Abnormal cervical cytology was seen more in lupus patients compared with controls $(16.4 \%$ vs. $2.8 \%, P<0.001)$ [2].

In the current study, no relationship between immunosuppressive medications and risk of HPV infection was found, because in the 2 HPV infected cases studied, one was on immunosuppressive medication, but the other was not. This was compatible with some other studies [6]. However, the limitation of a low number of patients is still there.

In the current study, patients had low SLEDAI $(0.44 \pm 1.07)$. A Korean study with a higher prevalence of HPV in their patients with lupus had a higher rate of SLEDAI with $8.1 \pm 6.4$ in their patients. Forty-four percent of patients with SLE in the current study had received immunosuppressive medications, but in $72.4 \%$ of Korean patients, immunosuppressive medications had been used because of higher disease activity. This may be a reason for the higher prevalence in their results. However, in the current research, there was no correlation between receiving immunosuppression and HPV infection or cervical dysplasia. The patients in the current study had one partner, but in the Korean study in which a high prevalence of HPV infection was seen, $13 \%$ of patients with SLE had more than one partner. This may be another interfering factor [2].

Another study done in 2015 compared 32 SLE patients with a normal population in Cairo, Egypt, and found HPV in only one patient. This result is comparable to the current study, but the Cairo study showed a higher prevalence of cervical changes in its patients compared to the normal population [13].

In a 2017 study in Brazil, researchers did not find any correlation with consumption of immunosuppressive medications in patients with SLE and other patients, although the prevalence of HPV infection was higher in the patients than in the normal population (20.8\% compared to $15 \%)$. Compared to the current study, the overall prevalence rate of HPV infection was higher [14].

Because of the low number of cases in the current study, future studies need to include more patients receiving immunosuppressive and new biologic medications to confirm the current results. Another limitation of the current study was that only married patients above 20 years of age were included. Future studies could include other groups of patients, too. It should be mentioned that it is important that women with lupus follow their regional guideline recommendations for cervical cancer screening.

\section{Conclusion}

Results of the current study showed that SLE and the consumption of immunosuppressive medications in patients with lupus are not risk factors for high-risk HPV infection or cervical dysplasia compared to the normal control population in our area, although there was a low number of patients. Further studies should investigate a greater number of patients with multiple risk factors receiving immunosuppressant and biologic medications due to the importance of future programs about the health of SLE patients.

\section{Conflicts of interest}

The authors declare no conflict of interest.

\section{Acknowledgments}

The present article was extracted from the thesis written by Dr. Poopak Mohaghegh and financially supported by Shiraz University of Medical Science, Grant number 916342. The authors would like to thank the Center for the Development of Clinical Research of Namazee Hospital and Dr. Nasrin Shokrpour for their editorial assistance. 


\section{References}

1. Tsokos GC. Systemic lupus erythematosus. The New England Journal of Medicine 2011; 365(22): 2110-21. Epub 2011/12/02. doi: 10.1056/NEJMra1100359.

2. Lee YH, Choe JY, Park SH, Park YW, Lee SS, Kang YM, et al. Prevalence of Human Papilloma Virus Infections and Cervical Cytological Abnormalities among Korean Women with Systemic Lupus Erythematosus. Journal of Korean Medical Science 2010; 25(10): 1431-7. doi: $10.3346 / \mathrm{jkms}$. 2010.25.10.1431.

3. Safaei A, Khanlari M, Momtahen M, Monabati A, Robati M, Amooei S, et al. Prevalence of high-risk human papillomavirus types 16 and 18 in healthy women with cytologically negative pap smear in Iran. Indian Journal of Pathology \& Microbiology 2010; 53(4): 681-5. Epub 2010/11/04. doi: 10.4103/ 0377-4929.72030

4. Bertsias G, Fanouriakis A, Boumpas DT. Treatment of systemic lupus erythematousus. In: Firestein G, Budd RC, Gabrieliain SE, James BM, O'Dell R, editors. Kelley's textbook of rheumathology. 2. 9 ed. Philadelphia: Elsevier; 2013: 130427.

5. Nath R, Mant C, Luxton J, Hughes G, Raju KS, Shepherd P, et al. High risk of human papillomavirus type 16 infections and of development of cervical squamous intraepithelial lesions in systemic lupus erythematosus patients. Arthritis and
Rheumatism 2007; 57(4): 619-25. Epub 2007/05/02. doi: 10.1002/art. 22667.

6. Santana IU, Gomes Ado N, Lyrio LD, Rios Grassi MF, Santiago MB. Systemic lupus erythematosus, human papillomavirus infection, cervical pre-malignant and malignant lesions: a systematic review. Clinical Rheumatology 2011; 30(5): 665-72. Epub 2010/11/13. doi: 10.1007/ s10067-010-1606-0.

7. Pellegrino P, Radice S, Clementi E. Immunogenicity and safety of the human papillomavirus vaccine in patients with autoimmune diseases: A systematic review. Vaccine 2015; 33(30): 3444-9. doi: 10.1016/j. vaccine.2015.05.041.

8. Dall'era M, Wofsy D. Clinical features of systemic lupus erythematosus. In: GF, Budd RC, Gabrielian SE, Mcinnes B, O'dell JR, editors. Kelley's textbook of rheumathology. 2. 9 ed. Philadelphia Elsevier; 2013.

9. Appleby P, Beral V, Berrington de Gonzalez A, Colin D, Franceschi S, Goodhill A, et al. Cervical cancer and hormonal contraceptives: collaborative reanalysis of individual data for 16,573 women with cervical cancer and 35,509 women without cervical cancer from 24 epidemiological studies. Lancet 2007; 370(9599): 1609-21. doi: 10.1016/s0140-6736(07)61684-5.

10. Smith JS, Bosetti C, Munoz N, Herrero R, Bosch FX, Eluf-Neto J, et al. Chlamydia trachomatis and invasive cervical cancer: a pooled analysis of the IARC multicentric case-control study. International Journal of Cancer Journal International du Cancer 2004; 111(3): 431-9. doi: 10.1002/ijc. 20257.

11. Shahramian I, Heidari Z, Mahmoudzadeh-Sagheb H, Moradi A, Forghani F. Prevalence of HPV infection and high risk HPV genotypes $(16,18)$, among monogamous and polygamous women, In Zabol, Iran. Iranian Journal of Public Health 2011; 40(3): 113-21.

12. Tam LS, Chan AY, Chan PK, Chang AR, Li EK. Increased prevalence of squamous intraepithelial lesions in systemic lupus erythematosus: association with human papillomavirus infection. Arthritis and Rheumatism 2004; 50(11): 3619-25. doi: 10.1002/art.20616.

13. Al-Sherbeni HH, Fahmy AM, Sherif N. Predisposition to cervical atypia in systemic lupus erythematosus: A clinical and cytopathological study. Autoimmune Diseases 2015; 751853. doi: 10.1155/2015/751853.

14. Amaral JL, Araujo MV, Dias GA, Ledebur EI, Quaresma JA, Fuzii HT. Clinical and epidemiological study of human papillomavirus infection in women with systemic lupus erythematosus in eastern brazilian amazon. Acta Reumatologica Portuguesa 2017; 42(1): 47-54. 